\title{
The effect of pictorial depth information on projected size judgments
}

\author{
EMILY K. FARRAN \\ Institute of Education, University of London, London, England
}

AND

Andrew Whitaker and Neha Patel

University of Reading, Reading, England

\begin{abstract}
When full depth cues are available, size judgments are dominated by physical size. However, with reduced depth cues, size judgments are influenced less by physical size and more by projected size. By manipulating monocularly presented pictorial depth cues only, in this study we reduced depth cues further than had previous size judgment studies. Participants were presented monocularly with two shapes against a background of zero (control), one, two, or three pictorial depth cues. Each cue was added progressively in the following order: height in the visual field, linear perspective, and texture gradient. Participants made a same/different judgment regarding the projected size of the two shapes (i.e., ignoring any depth cues). As was expected, accuracy increased and response times decreased as the ratio between the projected size of the two shapes increased (range of projected size ratios, 1:1-1:5). In addition, with the exception of the larger size ratios (1:4 and 1:5), detection of projected size difference grew poorer as depth cues were added. One- and two-cue conditions had the most weighting in this performance decrement, with little weighting from the three-cue condition. We conclude that even minimal depth information is difficult to inhibit, which indicates that depth perception requires little focused attention.
\end{abstract}

When they are available, depth cues combine to enable us to perceive the physical size of a static object in depth. This perception of physical size remains constant, despite changes in the distance of the object from the observer and the visual angle that it subtends (i.e., size constancy; see Sedgwick, 1986). In contrast to physical size, the projected size of an object is established by the visual angle that it subtends on the retina. When judgments of size are made, the relative influence of physical size and projected size is dependent on the amount of depth information available; that is, when depth is perceived, we expect a more distant object to have a smaller projected size, but to have the same physical size, than when it is closer in depth (Epstein, 1973). Depth can be determined by using the binocular cues of vergence and retinal disparity and by using monocular information, available through accommodation, motion parallax, and pictorial cues. We know that when adults are asked to make size judgments, such judgments are dominated by physical size over projected size, even in the absence of binocular cues, accommodation, and motion parallax - that is, when only pictorial cues are available (Uhlarik, Pringle, Jordan, \& Misceo, 1980; Yonas \& Hagen, 1973). However, little is known about the effect that reducing the number of pictorial cues has on the perception of size. In this study, our aim was to determine the point at which depth cues begin to influence size judgments - that is, how much depth information is required for the perception of size to be influenced by physical size information.

Yonas and Hagen (1973) investigated depth perception by manipulating accommodation (3-D vs. 2-D presentation) and motion parallax (present vs. absent) depth cues in a size judgment task. Two real triangles of different physical size, presented monocularly, were positioned in a real textured alley, and participants were asked which triangle was larger. The visual angle (projected size) subtended by the large triangle either was equal to that subtended by the small triangle or was $70 \%$ or $80 \%$ of the projected size of the smaller triangle. Adult participants used the available cues and gave judgments according to physical size. Three- and 7-year-olds responded to physical size when the projected size of the two triangles was equal. However, when the projected and physical size differences were incongruent, children required at least 3-D depth information in order to respond according to physical size, and on the hardest trials ( $70 \%$ projected size difference), 3-year-olds also required the additional depth cue of motion parallax. These results indicate that, with development, perception of physical size is possible with progressively fewer depth cues, and, for adults, pictorial cues alone are sufficient for physical size to dominate. The present study further investigates this dominance by testing with one, two, and three pictorial cues. 
Uhlarik et al. (1980) demonstrated that pictorial depth cues affect size judgments, even when participants are asked to respond according to projected size. Participants viewed a 2-D photograph of a shape placed in a textured 3-D alley under binocular viewing conditions. Participants were explicitly asked to judge the physical size (referred to as "objective size") or the projected size of a shape, in units, relative to a standard sized shape. Although physical size judgments generally were accurate, projected size judgments were distorted by depth information; projected size responses were almost halfway between those predicted by physical and by projected size. This demonstrates that depth cues cannot be inhibited completely for projected size judgments and suggests that at least some depth information is processed automatically. However, because numerous pictorial cues remained present, we do not know the extent of this distorting effect. In the present study, we ask whether distortion remains with reduced pictorial cues, and whether the strength of the bias is influenced by the type or number of depth cues available.

The influence of depth cues has also been demonstrated in studies of visual search. Visual search performance is affected by depth information, such as 3-D orientation (Enns \& Rensink, 1991), direction of light in a scene (Aks \& Enns, 1992), and texture gradient (Aks \& Enns, 1996). Therefore, it appears that, in adults, depth perception is a relatively low-level process that occurs in the early stages of visual perception. That is, in common with some visual search mechanisms, depth cues are processed preattentively (see Treisman, 1986) and, therefore, are difficult to inhibit (Uhlarik et al., 1980).

Bennett and Warren (2002) adapted a standard size transformation task to investigate the influences of projected and physical size on performance. Standard size transformation tasks (e.g., Bundesen \& Larsen, 1975; Howard \& Kerst, 1978; Jolicœur \& Besner, 1987) differ from the size judgment tasks described above. A size transformation task is a mental imagery task. Participants are shown two nonuniform objects that differ in size and also, on $50 \%$ of trials, differ subtly in form. Participants must determine whether the two objects are the same or different in form, ignoring any differences in size. Successful completion is thought to rely on participants' mental ability to scale one object to match the size of the other, thus enabling them to make a same/different judgment of form alone; a linear increase in response time (RT) is observed with increased size ratio between the two objects.

In standard size transformation tasks, objects are presented in the same depth plane, with no background depth information. As such, projected size and physical size are equivalent, and their relative influence on performance cannot be differentiated. Introducing progressive amounts of depth information enabled Bennett and Warren (2002) to investigate the relative influence of projected and physical size on participants' judgments of form in their size transformation study. Displays were viewed monocularly from behind a reduction screen, and depth information decreased across three conditions: a textured corridor with shadow information, a textured corridor with no shadow, and a vertical textured wall (forms still could differ in height in the picture plane). Stimuli were presented at different simulated distances, with five projected and physical size ratios (range, 1:1-1:3). Results demonstrated significant linear increases in RT for both physical and projected size ratios in all conditions. The authors suggested that, before mental size scaling and, thus, form matching proceeds, size is coded by pooling all available size information. For both of the 3-D "corridor" conditions, the linear increase in RT was comparable across physical and projected size ratios. This suggests that the influence of projected and physical size was roughly equal. Such a result is surprising, given that size judgment studies have reported physical size dominance in similar conditions. This could relate to additional projected-size information from the flat screen (accommodation, motion parallax), as suggested by Bennett and Warren, or it could be related to the difference in task demands (form vs. size judgment). Performance on the 2-D condition, however, showed a stronger influence of projected than physical size. Importantly, this indicates that manipulating pictorial depth cues influences the relative influence of physical and projected size on form judgments and that, with only very minimal cues, projected size dominates. This is investigated further in the present study by using a size judgment task. To our knowledge, this is the first study to investigate the effect of manipulating pictorial depth cues on same/different judgments of projected size.

In summary, previous studies have shown that, with reduced depth cues and provided there are numerous pictorial depth cues, physical size remains dominant over projected size (Uhlarik et al., 1980; Yonas \& Hagen, 1973). However, Bennett and Warren (2002) provided evidence to suggest that projected size might dominate if pictorial cues are also reduced. To investigate this, we asked participants to make size judgments with reduced pictorial cues only. Stimuli were viewed monocularly, in an environment in which objects rested on the ground plane (the ground plane was flat and the horizon was at eye level). The first cue added was height in the visual field (Wallach \& O'Leary, 1982). For this cue, the angle between eye level (assuming that this is at the horizon) and the location of the base of the object (assuming that this is below eye level) determine the distance of the object from the observer: Objects that are farther away appear higher in the visual scene and, thus, subtend a smaller angle. The second depth cue added was linear perspective, which Sedgwick (1986) explains indicates size and depth in two ways. First, as a direct indication of size (see Wraga, 1999), there is a constant relationship between the height of an object resting on a surface and the vertical distance between eye level (assuming that this is at the horizon) and that surface. From this, the ratio between the visual angles subtended from the point of observation between the top and bottom of the object and between the horizon and bottom of the object can be calculated; in Sedgwick (1973), this is referred to as the horizon-ratio relationship. The second way in which linear perspective indicates size and depth is also specified by the third depth cue added, texture gradient. For both linear perspective and texture gradient, depth is indicated by the angular separation between the projection of converging horizontal surface lines (edges or 
texture), which provide a constant scaling factor toward the horizon (Gibson, 1950; Sedgwick, 1986).

For each condition, participants were explicitly asked to make same/different size judgments according to projected size only. The influence of physical size was determined by the negative effect on performance, relative to the control condition where no depth cues were available. Our aim was to determine both when depth cues begin to affect projected size judgments and the relative weighting of each of the depth cues employed.

\section{METHOD}

\section{Participants}

Forty participants, 22 female and 18 male, from 18 to 29 years of age $(M=19.43$ years; $S D=0.29)$ were recruited. Participants were undergraduate students at the University of Reading. All had normal or corrected-to-normal vision. Twenty-two $(55 \%)$ participants were left-eye dominant and 18 (45\%) were right-eye dominant (45\%).

\section{Eye Dominance Tasks}

Eye dominance was determined by employing two methods cited in Roth, Lora, and Heilman (2002). The first was a variation of the Porta test (della Porta, 1593): Participants were asked to extend one arm and to align their index finger vertically with the corner of the testing room, with both eyes open. Participants then had to close one eye at a time and report which eye closure caused the least change in the alignment of their index finger with the corner of the wall. The eye reported was recorded as the dominant eye. The second test was a variation of the Miles test (Miles, 1930). Participants were asked to make a small hole between both of their extended hands and to view a small object (a piece of metal) through the hole. They were then asked to move their hands toward their face, while fixating on the object in the hole. The eye that participants brought their hands nearest to was recorded as the dominant eye. All participants showed the same eye dominance for both tests.

\section{Experimental Task}

Design. Viewing was made monocular by asking participants to wear an eye patch over their nondominant eye. Viewing distance from the screen was controlled by using a chinrest, fixed at $39.10 \mathrm{~cm}$ from a 33.00-cm-wide and 21.00-cm-high screen (resolution: 1,920 × 1,200 pixels). Thus, the screen subtended a visual angle of $45.76^{\circ} \times 30.06^{\circ}$.

Each image depicted two shape stimuli (either two black triangles or two black squares; see Figure 1) on a background of zero to three monocular depth cues (created using Paint Shop Pro, v. 7.0, and Alice, v. 2.0). Control trials contained no depth information. One-cue trials employed the depth cue of height in the visual field. Two-cue trials employed height in the visual field and linear perspective. Three-cue trials used height in the visual field, linear perspective, and texture gradient. The texture employed depicted a typical concrete-type surface (surface.bmp from CorelDRAW 9). Five projected shape sizes were employed, with an area of $1,2,3,4$, and $5 \mathrm{~cm}^{2}$, respectively. The left-hand shape was positioned at $x=75 \mathrm{~mm}, y=30 \mathrm{~mm}$, throughout. The righthand shape was positioned at $x=175 \mathrm{~mm}, y=30 \mathrm{~mm}$, for control trials, and at $x=175 \mathrm{~mm}, y=185 \mathrm{~mm}$, for depth-cue trials. The two presented shapes were either the same projected size or different projected sizes ( $50 \%$ of trials for each). Because these corresponded to participants' correct responses of "same" or "different," respectively, corresponding trials will henceforth be referred to as same or different trials. There were 10 size combinations of different-sized
A

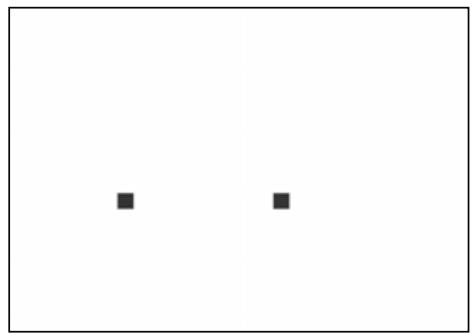

Condition: control (no cue)

Projected size ratio: $1: 1$

Physical size ratio: 1:1

C

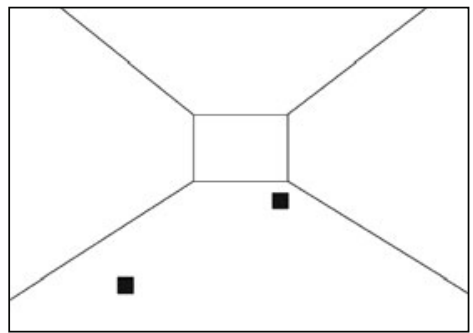

Condition: two-cue (height in the visual field, linear perspective)

Projected size ratio: 1:1

Physical size ratio: 1:2.57
B

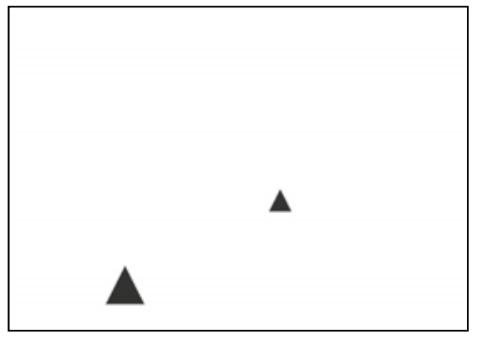

Condition: one-cue (height in the visual field)

Projected size ratio: $3: 1$

Physical size ratio: 1:1.48

D

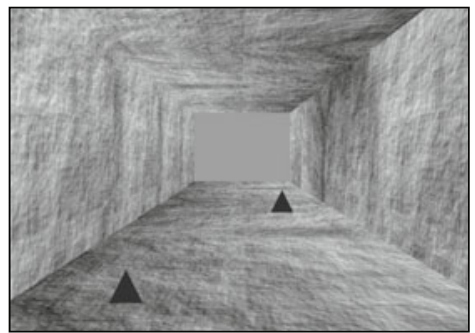

Condition: three-cue (height in the

visual field, linear perspective, texture)

Projected size ratio: 2:1

Physical size ratio: 1:1.82

Figure 1. Example stimulus images. 
Table 1

Corresponding Projected and Physical Size Ratios for Depth-Cue Trials

\begin{tabular}{|c|c|c|c|c|c|c|c|c|c|c|}
\hline & \multicolumn{10}{|c|}{ Size Ratio } \\
\hline $0{ }^{2}$ & $1: 1$ & $1.25: 1$ & $1.33: 1$ & $1.5: 1$ & $1.67: 1$ & $2: 1$ & $2.5: 1$ & $3: 1$ & $4: 1$ & $5: 1$ \\
\hline Physical & $1: 2.57$ & $1: 2.30$ & $1: 2.23$ & $1: 2.10$ & $1: 1.99$ & $1: 1.82$ & $1: 1.63$ & $1: 1.48$ & $1: 1.28$ & $1: 1.15$ \\
\hline
\end{tabular}

shapes $\left(2: 1,3: 1,4: 1,5: 1,3: 2,4: 2,5: 2,4: 3,5: 3,5: 4 \mathrm{~cm}^{2}\right)$ and 5 possible combinations of same-sized shapes $\left(1: 1,2: 2,3: 3,4: 4,5: 5 \mathrm{~cm}^{2}\right)$. To give equal numbers of same and different trials, same trials were presented twice, for each shape, in each condition. The projected size ratio of these shapes varied from 1:1 (same trials) to $5: 1\left(5: 1 \mathrm{~cm}^{2}\right)$. In different trials, the projected size of the left-hand shape was larger than the right-hand shape.

The center of the display was at each participant's eye level, and therefore the vertical midpoint of the back wall of the alley equated to where a true horizon would be. If depth was judged from the height of the object in the scene, this equates to the angular subtense of each object at the eye. The angular subtense can be calculated from the vertical distance between the "horizon" and the base of each object (left shape, $9 \mathrm{~cm}$; right shape, $3.5 \mathrm{~cm}$ ), and the distance of the observer from the screen $(39.1 \mathrm{~cm})$. Physical size ratio calculations were based on the ratio between these vertical distances (and thus the visual angle that they subtend), which was $1: 2.57(9.00 \mathrm{~cm} / 3.5 \mathrm{~cm})$. Thus, where $\tan \theta 1$ and $\tan \theta 2$ are calculated from the projected size of the object on the left (physically near) and the projected size of the object on the right (physically far), respectively, the physical size ratio is $1: x$, where $x=2.57 \tan \theta 2 / \tan \theta 1$. Corresponding projected and physical size ratios are shown in Table 1 . Physical size ratio varied from $1: 2.57$ to $1: 1.15$. There were 160 experimental trials in total: four monocular cue conditions, each with 40 trials (10 same, 10 different for each of the two shapes [square, triangle]).

Procedure. Participants were informed that the study was concerned with how certain depth cues affect one's perception of the size of an object. They were instructed to judge whether the size of two objects was the same or different, while ignoring the depth cues. They responded by pressing one of two keys labeled "same" and "different." Participants were told that there was no time limit, but that RTs would be recorded. Participant responses were followed by a 200-msec interstimulus interval and a 200-msec fixation mask before the next trial began. Participants took part in a practice block of 8 trials to ensure that they understood the procedure. This comprised 2 existing trials drawn from each of the four cue conditions. Fol- lowing this, the 160 experimental trials were presented in a random order. Feedback was given on the practice trials only.

\section{RESULTS}

\section{Projected and Physical Size Ratios}

"Same" and "different" responses were recorded and the proportions of "different" responses analyzed. For the projected size ratio of $1: 1$, a "different" response corresponds to an incorrect response, and for projected size ratios from 1.25:1 to 5:1, a "different" response is correct.

The data set was not distributed normally for projected size ratios from 1.25:1 to 5:1 (Kolmogorov-Smirnoff, $p<$ .05 for all) and so all data underwent arcsine transformation before analysis. A two-way ANOVA of proportion of "different" responses was carried out, with monocular cue (four conditions: control, one-cue, two-cue, three-cue) and projected size ratio (10 levels: $1: 1,1.25: 1,1.33: 1,1.5: 1,1.67: 1$, $2: 1,2.5: 1,3: 1,4: 1,5: 1)$ as factors. The main effects of monocular cue [linear contrast, $F(1,39)=83.17, p<.001, \eta_{\mathrm{p}}^{2}=$ .68 ] and projected size ratio [linear contrast, $F(1,39)=$ $\left.281.89, p<.001, \eta_{\mathrm{p}}^{2}=.88\right]$ were mediated by an interaction between these two factors $[F(27,1053)=25.97, p<$ $\left..001, \eta_{\mathrm{p}}^{2}=.40\right]$. This is illustrated in Figure 2, which shows that, when a "different" response was a correct response (all projected size ratios, except 1:1), for small size ratios, as the number of monocular cues increased it became increasingly more difficult to determine that the projected sizes of the two shapes were different (ratios of 1.25:1 to $3: 1, p<.001$ for all). This was not the case for the larger projected size ratios, where the proportion of "different" responses was

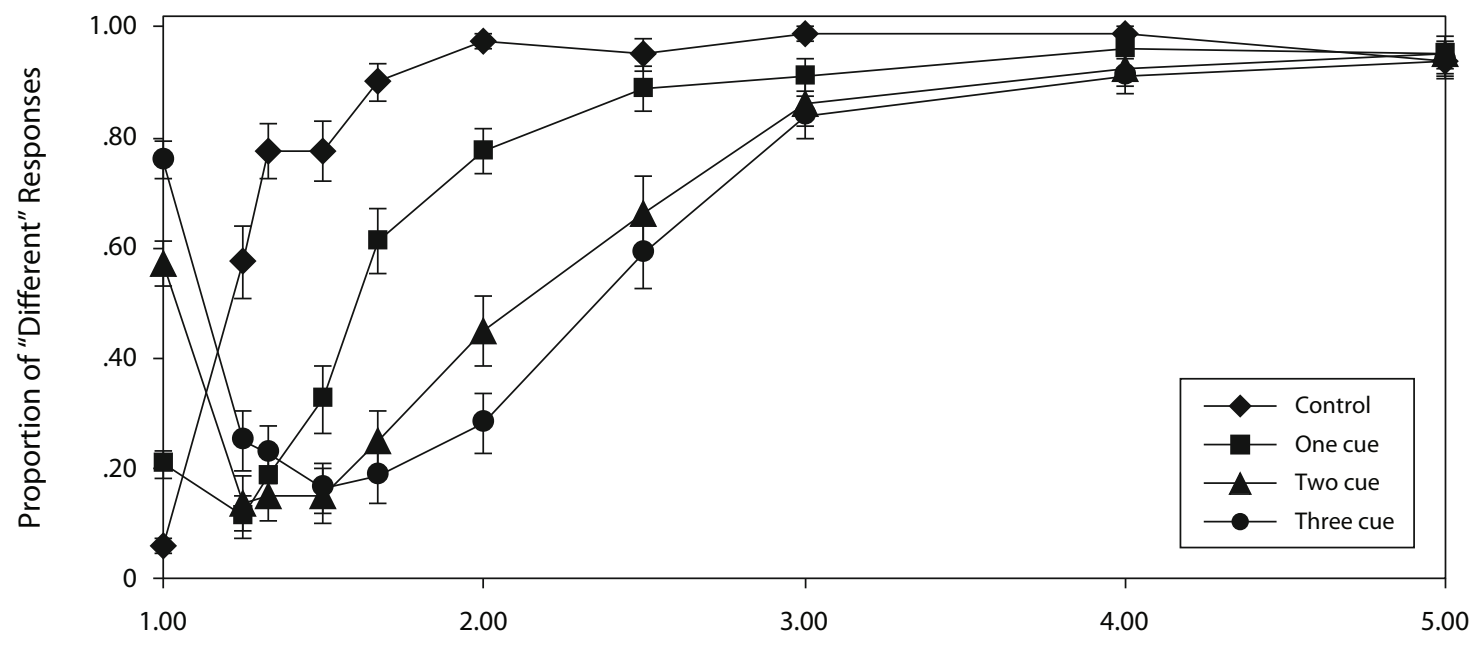

Projected Size Ratio of $x: 1$

Figure 2. Proportion of "different" responses for projected size judgments at each cue condition. 
high across all monocular cue conditions $[4: 1, F(3,117)=$ $\left.2.02, p=.11, \eta_{\mathrm{p}}^{2}=.01 ; 5: 1, F<1\right]$. When a "different" response was an incorrect response (a projected size ratio of $1: 1)$, the pattern of responses differed from other projected size ratios. For the control, no-cue condition, participants were, as was expected, unlikely to incorrectly report a size difference. Similarly, only a small proportion of "different" responses were given in the one-cue condition, which was in line with the steady increase in the proportion of "different" responses across increases in projected size ratio. However, for the two- and three-cue conditions, the proportion of "different" responses was high, and was not in line with the trend in "different" responses across increasing projected size ratios. This suggests that there is something
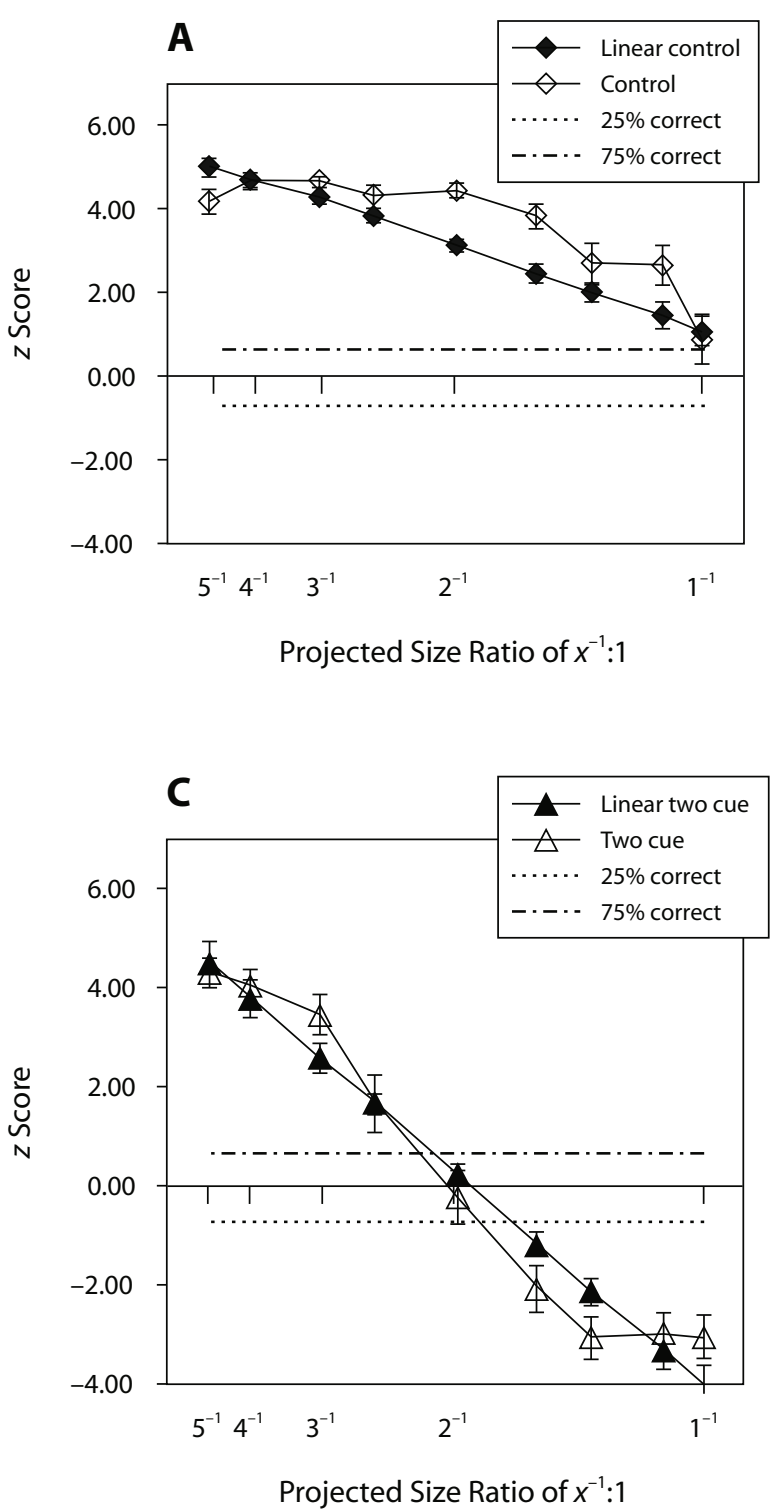

special about same trials that differentiates them from different trials. This differentiation will be examined in the Discussion section.

Further analysis determined the point at which a projected size difference could no longer be detected reliably. As was described above, a response of "different" was an incorrect response for same trials ( $1: 1$ projected ratio), but a response of "different" was a correct response for different trials (1.25:1 to 5:1 projected ratios). Because we were interested in the ability to detect only projected size difference, same trials were not included in this analysis. For each condition, the projected size ratio at which "different" responses were given on $25 \%$ of trials was employed as a threshold. This is the $75 \%$ threshold for incorrect (nonprojected) responses
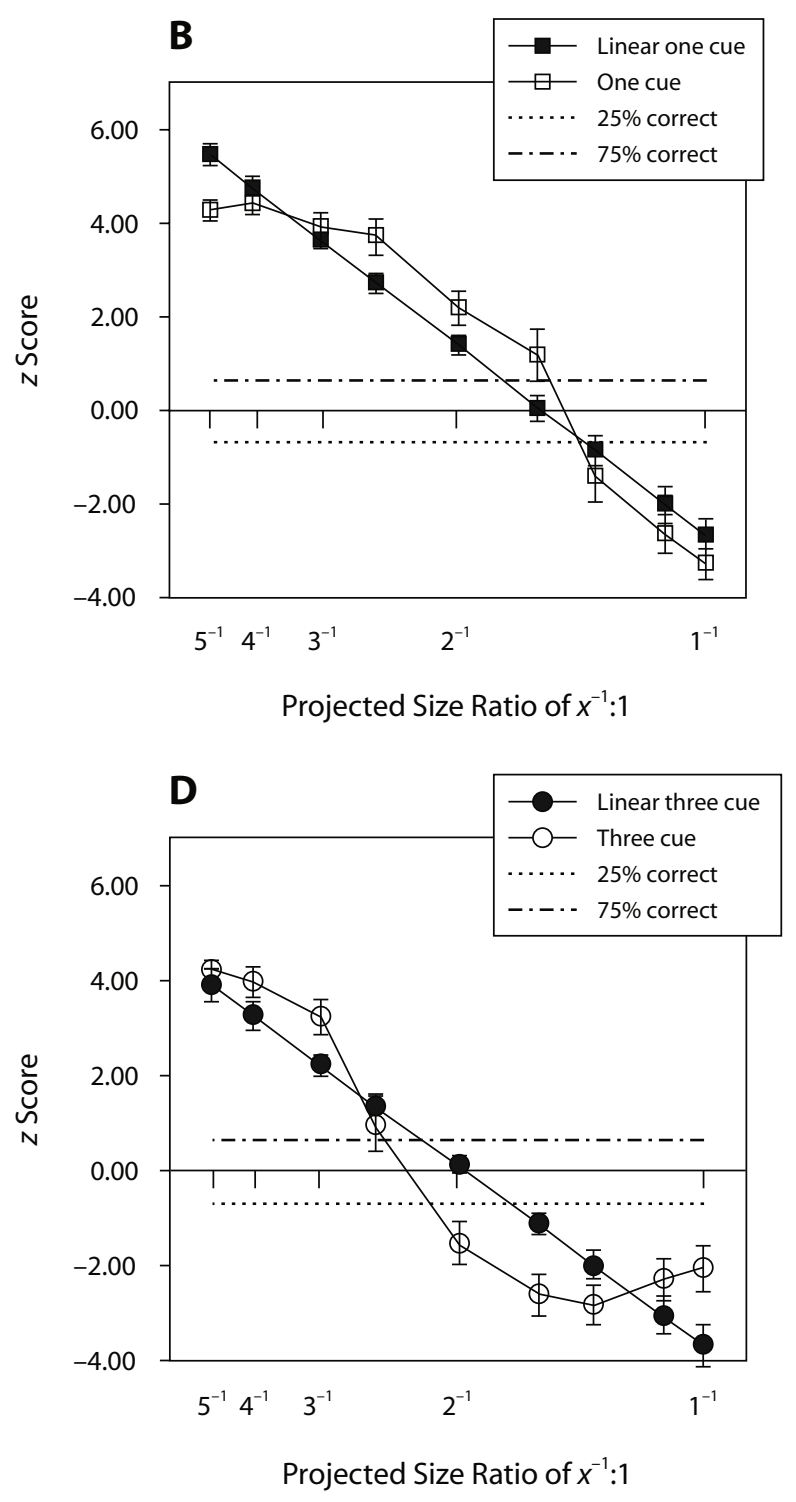

Figure 3. Actual mean $z$ scores of proportion of "different" responses, plotted against transformed projected size judgments $\left(x^{-1}\right.$ :1) for the control condition (A), the one-cue condition (B), the two-cue condition (C), and the three-cue condition (D). Open shapes show mean participant $z$ scores, plotted for each transformed projected size judgment. Closed shapes indicate the mean participant linear function between $z$ scores and transformed projected size judgments. Linear functions were employed to determine the weighting of each depth cue. 


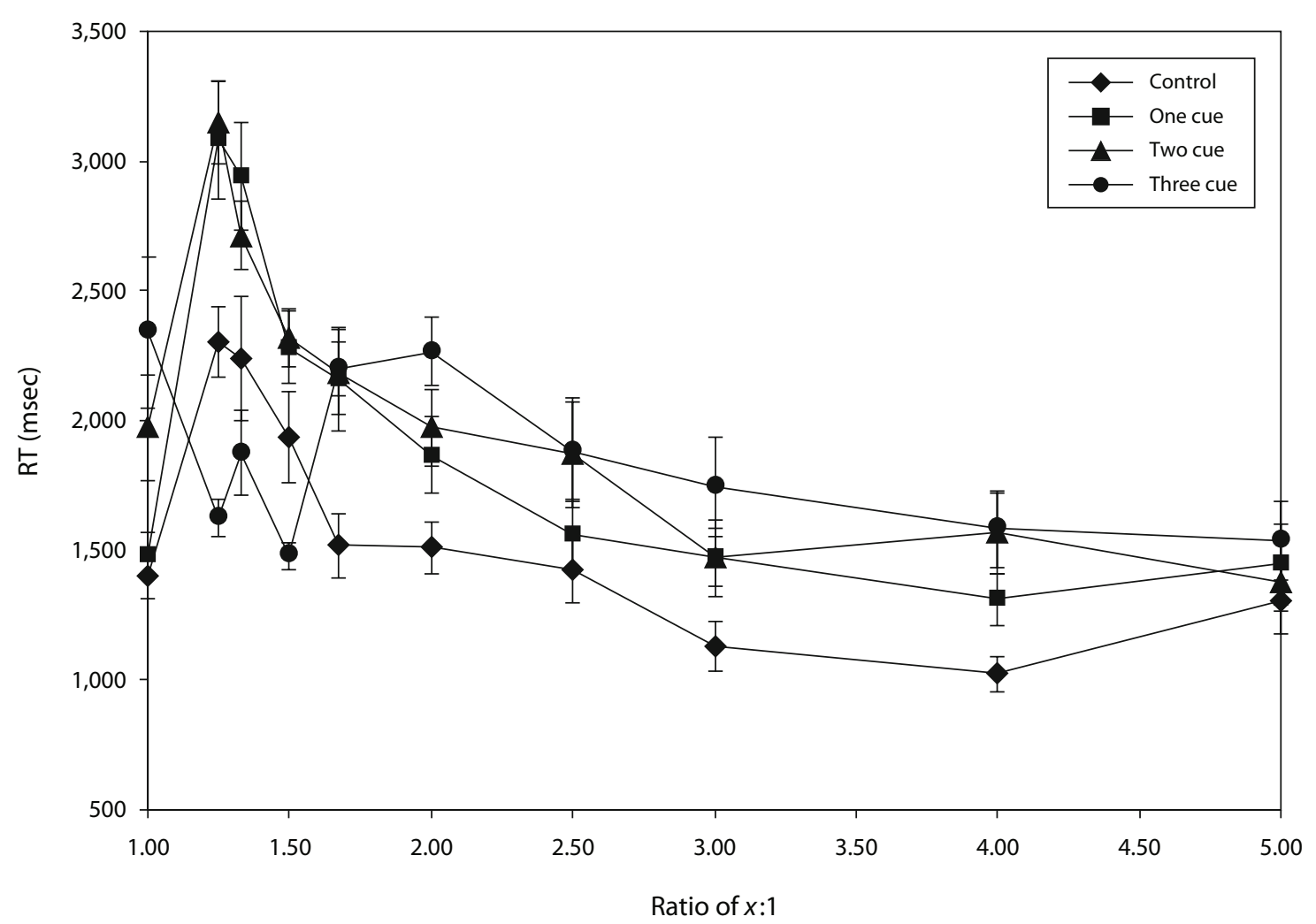

Figure 4. Response times (RTs) for projected size judgments.

and, thus, the threshold at which the influence of depth information is too strong to inhibit. Proportion of "different" responses was converted to $z$ scores and plotted against $x^{-1}$, where $x$ represents an $x: 1$ projected size ratio. Using $x^{-1}$ transforms performance on each condition into a straight line [linear regression of mean $z$ scores of each cue condition against $x^{-1}$ : control condition, $F(1,7)=16.844, p=$ .01 ; one-cue condition, $F(1,7)=90.33, p<.001$; two-cue condition, $F(1,7)=139.24, p<.001$; three-cue condition, $F(1,7)=40.06, p<.001]$. Individual linear functions of the $z$ score against $x^{-1}$ were calculated for each participant for each condition. The mean of these functions is plotted in Figure 3, alongside the actual mean $z$ scores, to illustrate the linear fit. From each linear function, each participant's $75 \%$ threshold ( $25 \%$ correct), at which depth information could not be inhibited, was determined for each depth condition. Thresholds of correct responses of $75 \%$ and $25 \%$ are illustrated in Figure 3. This shows that performance on the control condition was always above $75 \%$; thus, differences can be detected from at least a projected ratio of 1.25:1. For cue conditions, projected size differences could be detected from a ratio of 1.36:1 with one cue, 1.79:1 with two cues, and 1.82:1 with three cues.

We also calculated the cumulative weighting of each cue on the ability to judge projected size. To understand how this was calculated, consider the hardest different trials - that is, where the projected size ratio is the smallest, 1.25:1. On these trials, if depth cues could not be inhibited at all, the participant would perceive physical size only, and the right-hand object would appear 2.30 times larger than the left-hand ob- ject (see Table 1). Thus, we calculated each individual's 75\% threshold projected size ratio (i.e., the point at which physical size differences could not be inhibited) as a proportion of 2.30 (100\% influence). This showed that depth information was weighted at $67.47 \%(20.83 \%), 80.25 \%(13.59 \%)$, and $87.31 \%(40.16 \%)$ for one-, two-, and three-cue conditions, respectively. This corresponded to significantly higher weightings for two and three cues, as compared with one cue ( $p<.001$ for both), but no difference between the weightings toward two- and three-depth cues $(p=.23)$.

\section{Response Times}

Only RTs for correct responses were analyzed. RT data are illustrated in Figure 4 and are consistent with the proportion correct data. As with the proportion correct data, a two-way ANOVA was carried out with monocular cue and projected size ratio as factors. Consistent with the proportion correct data, the main effects of monocular cue $\left[F(3,117)=21.17, p<.001, \eta_{\mathrm{p}}^{2}=.35\right]$ and projected size ratio $\left[F(1,39)=95.27, p<.001, \eta_{\mathrm{p}}^{2}=.71\right]$ were mediated by an interaction between these two factors $\left[F(27,1053)=6.62, p<.001, \eta_{\mathrm{p}}^{2}=.15\right]$. This was because of differences in the main effect of cue as the projected size ratio increased. For size ratios of $1: 1$ to $2: 1$, $3: 1$, and $4: 1$, responses were faster the fewer cues there were $(1: 1-1.67: 1,4: 1, p<.001 ; 2: 1,3: 1, p<.05)$; for the size ratio of $2.5: 1$, this main effect was marginally significant $(p=.06)$, whereas for the largest size ratio of $5: 1$, the number of cues present had no significant effect on $\operatorname{RTs}(p=.55)$. 


\section{DISCUSSION}

We were interested in the level of monocular pictorial depth information required to affect the ability to perceive the projected size of an object. The results demonstrate that participants were able to respond to projected size as requested, rather than physical size ratios: As projected size ratio increased, performance became more accurate and responses became quicker. The main effect of monocular cues, however, clearly demonstrates that it was effortful to inhibit the influence of depth cues. Relative to the nocue control condition, performance became increasingly less accurate and responses slower, with the addition of each depth cue. Even for the single monocular depth cue of height in the visual field, physical size had to be inhibited actively in order for participants to respond in line with projected size. This finding is consistent with, and expands on, the results of Uhlarik et al. (1980) and Yonas and Hagen (1973), who showed a dominance of physical over projected information with numerous pictorial depth cues.

We also estimated the threshold at which a difference in projected size could no longer be detected accurately because of sensitivity to depth information. On the control (no-cue) trials, small projected size ratios were relatively difficult to detect, but accuracy was consistently above the $75 \%$ level of performance. This was not the case for trials that included depth cues. Participants could reliably detect a projected size difference only from a projected size ratio of 1.36:1 for one-cue trials, 1.79:1 for two-cue trials, and 1.82:1 for three-cue trials. Below these ratios, the interference from depth cues was great enough to reduce detection of projected size difference to below $25 \%$. The addition of each depth cue was not equally detrimental. Adding the height cue in the visual field strongly influenced performance. There was also a substantial influence of adding linear perspective as a cue. At this point, sensitivity to depth cues showed a plateau: The inclusion of texture gradient as a depth cue had little additional effect on performance.

Cues were added in a fixed order; thus, one cannot determine the extent to which the weightings reflect the number of cues available. Also, studies have shown that the reliability of an individual cue changes according to such factors as viewing distance and the slant of a surface and that this reliability dictates the relative influence of each cue (e.g., Bradshaw, Glennerster, \& Rogers, 1996; Ernst \& Banks, 2002; Hillis, Watt, Landy, \& Banks, 2004). As such, the weightings of the three cues observed in this study were not fixed and could vary with differences in viewing distance or if slant was introduced to the flat ground plane.

It is possible that the relatively small additional weighting observed for texture gradient, relative to the two-cue condition, relates to the nature of the texture, a concretetype texture. Texture gradient is not a singular depth cue, since it involves the gradients of size, density, and compression. The texture employed clearly depicted a receding texture with size, density, and compression gradients; however, it did not have any defined texture units. This was intentional, since we did not want the depth cue of relative size to govern performance (see Cutting \& Vishton, 1995; Gillam, 1995). However, it is possible that a texture with visible texture units might have commanded a higher weighting on performance. Participants might have shown more interference, since size would be measured against the size of texture units.

Overall, it appears that with only two pictorial cues, performance is heavily influenced by physical size information despite instructions to ignore this information. This suggests that below a certain projected size ratio, depth cues cannot be ignored, which supports the suggestion that depth information is perceived at a preattentive level (Aks \& Enns, 1992, 1996; Enns \& Rensink, 1991).

The effect of monocular depth diminished as the projected size ratio between shapes increased. Indeed, when the projected size ratio was 5:1, there was no effect of cue for either proportion of "different" responses or RT. This was also true for proportion of "different" responses when the projected size ratio was $4: 1$. Performance on these trials was no different regardless of whether there were no depth cues or all three depth cues were present. Thus, given a large enough projected difference in size, conflicting information from depth cues is relatively inconsequential and can be ignored.

For same trials, the effect of two and three cues was out of line with responses to different trials in these conditions. One might argue that this result reflects the relationship between projected and physical size ratios. The 1:1 projected size ratio trials represented the largest physical size ratio, and thus it is possible that these trials were the most likely to elicit a "different" response, if the judgment was driven strongly by physical size. However, if this were the case, one would predict an interaction between increasing projected size difference and decreasing physical size difference, which would produce a U-shaped function for the two- and three-cue conditions. Inspection of Figure 2 does not support this; performance at a projected size ratio of $1: 1$ is distinctly different from the pattern of performance for the other ratios. Thus, we conclude that there is something special about the influence of depth cues on performance, when the projected size of two objects is identical. If that is so, it appears that participants do treat identical projected size trials differently from other trials, which suggests some subconscious awareness, but that this results in a stronger influence of conflicting depth information, rather than the reduced influence that one might predict. Data from previous studies shed little light on this effect. Yonas and Hagen (1973) did not specify that participants judge projected size, and Bennett and Warren's (2002) task was a size transformation (form judgment), rather than a size judgment task. Uhlarik et al. (1980) did ask participants to judge projected size and, in contrast to the present findings, showed that responses to a projected size ratio of 1:1 were in line with the trend across projected size ratios. Participants were asked to judge the size of one block, in units, relative to another block. Because this type of responding is along a continuum, perhaps the binary, same/different, responding required in the present study was the root of the unusual effect for same trials. This could be determined through further investigation of "different" response types. 
In this study, depth was manipulated at the level of pictorial monocular depth cues. As such, the amount of depth information available was reduced relative to previous size judgment studies, insofar as both Uhlarik et al. (1980) and Yonas and Hagen (1973) included numerous pictorial monocular depth cues across all depth conditions. By asking adult participants specifically to make judgments of projected size, we revealed a differentiation in the extent to which depth information affects size judgments. It appears that even one pictorial depth cue affects a projected size response and that this effect is cumulative with depth cues, with a plateau at two cues. Previous studies of size judgment did not show this differentiation, since the lowest cue condition already contained the ceiling amount of cues to encourage the complete dominance of physical size ratios over projected size ratios in adults.

As in the present study, pictorial depth cues were manipulated in the form judgment study employed by Bennett and Warren (2002). Their pattern of results was similar to the pattern observed here, with a stronger influence of physical size for texture and height in the visual field, as compared with the latter cue alone. However, projected size remained influential across conditions; thus, the extent of effect was attenuated, as compared with the results in the present study. Comparison between their form judgment study and our size judgment study suggests that physical size is easier to inhibit in a form judgment task than in a size judgment task, although differences in the influence of the flat presentation screen cannot be ruled out.

In summary, even minimal depth information has some influence on projected size judgments in adulthood, provided that the size judgments are sufficiently difficult. These results illustrate the preattentive nature of depth processing, even at the level of pictorial monocular cues.

\section{AUTHOR NOTE}

The authors thank John Wann, Richard Wilkie, and David Field for helpful comments on the manuscript. The authors also thank Richard Whitaker for providing an efficient data collapsing procedure. Correspondence concerning this article should be addressed to E. K. Farran, Department of Psychology and Human Development, Institute of Education, 25 Woburn Square, London WC1H 0AA, England (e-mail: e.farran@ioe.ac.uk).

Note-Accepted by the previous editorial team, when Thomas H. Carr was Editor.

\section{REFERENCES}

AKs, D. J., \& ENNS, J. T. (1992). Visual search for direction of shading is influenced by apparent depth. Perception \& Psychophysics, 52, 63-74.

AKs, D. J., \& EnNs, J. T. (1996). Visual search for size is influenced by a background texture gradient. Journal of Experimental Psychology: Human Perception \& Performance, 22, 1467-1481.

Bennett, D. J., \& WARren, W. (2002). Size scaling: Retinal or environmental frame of reference? Perception \& Psychophysics, 64, 462-477.

Bradshaw, M. F., Glennerster, A., \& Rogers, B. J. (1996). The effect of display size on disparity scaling from differential perspective and vergence cues. Vision Research, 36, 1255-1264.

Bundesen, C., \& Larsen, A. (1975). Visual transformation of size. Journal of Experimental Psychology: Human Perception \& Performance, 1, 214-220.

Cutting, J. E., \& Vishton, P. M. (1995). Perceiving layout and knowing distances: The integration, relative potency, and contextual use of different information about depth. In W. Epstein \& S. Rogers (Eds.), Handbook of perception and cognition: Vol. 5. Perception of space and motion (pp. 69-117). San Diego: Academic Press.

Della PoRTA, G. (1593). De refractione optices parte: Libri nouem. Naples: Iacobum Carlinum \& Antonium Pacem.

Enns, J. T., \& Rensink, R. A. (1991). Preattentive recovery of threedimensional orientation from line drawings. Psychological Review, 98, 335-351.

Epstein, W. (1973). The process of "taking-into-account" in visual perception. Perception, 2, 267-285.

ERnst, M. O., \& Banks, M. S. (2002). Humans integrate visual and haptic information in a statistically optimal fashion. Nature, 415, 429433.

GiBson, J. J. (1950). The perception of the visual world. Boston: Houghton Mifflin.

Gillam, B. (1995). The perception of spatial layout from static optical information. In W. Epstein \& S. Rogers (Eds.), Handbook of perception and cognition: Vol. 5. Perception of space and motion (pp. 23-67). San Diego: Academic Press.

Hillis, J. M., WatT, S. J., Landy, M. S., \& BanKs, M. S. (2004). Slant from texture and disparity cues: Optimal cue combination. Journal of Vision, 4, 967-992.

HowaRd, J. H., \& KERST, S. M. (1978). Directional effects of size change on the comparison of visual shapes. American Journal of Psychology, 91, 491-499.

Joliceur, P., \& BesNer, D. (1987). Additivity and interaction between size ratio and response category in the comparison of size-discrepant shapes. Journal of Experimental Psychology: Human Perception \& Performance, 13, 478-487.

MiLes, W. R. (1930). Ocular dominance in human adults. Journal of General Psychology, 3, 412-430.

Roth, H. L., Lora, A. N., \& Heilman, K. M. (2002). Effects of monocular viewing and eye dominance on spatial attention. Brain, 125, 2023-2035.

SEDGWICK, H. A. (1973). The visible horizon: A potential source of visual information for the perception of size and distance. Dissertation Abstracts International, 34, 1301B-1302B (UMI No. 73-22, 530).

SedGwick, H. A. (1986). Space perception. In K. R. Boff, L. Kaufman, \& J. P. Thomas (Eds.), Handbook of perception and human performance: Vol. 1. Sensory processes and perception (pp. 21.1-21.57). New York: Wiley.

Treisman, A. (1986). Properties, parts, and objects. In K. R. Boff, L. Kaufman, \& J. P. Thomas (Eds.), Handbook of perception and human performance: Vol. 2. Cognitive processes and performance (pp. 35.1-35.70). New York: Wiley.

Uhlarik, J., Pringle, R., Jordan, K., \& Misceo, G. (1980). Size scaling in two-dimensional pictorial arrays. Perception \& Psychophysics, 27, 60-70.

Wallach, H., \& O'Leary, A. (1982). Slope of regard as a distance cue. Perception \& Psychophysics, 31, 145-148.

Wraga, M. (1999). The role of eye height in perceiving affordances and object dimensions. Perception \& Psychophysics, 61, 490-507.

Yonas, A., \& HAGEN, M. (1973). Effects of static and motion parallax depth information on perception of size in children and adults. Journal of Experimental Child Psychology, 15, 254-265.

(Manuscript received July 12, 2005; revision accepted for publication August 25, 2008.) 